\title{
Multivariate Linkage Analysis of Specific Language Impairment (SLI)
}

\author{
Anthony P. Monaco* \\ THE SLI CONSORTIUM (SLIC) ${ }^{1}$
}

\section{SUMMARY}

Specific language impairment (SLI) is defined as an inability to develop appropriate language skills without explanatory medical conditions, low intelligence or lack of opportunity. Previously, a genome scan of 98 families affected by SLI was completed by the SLI Consortium, resulting in the identification of two quantitative trait loci (QTL) on chromosomes 16q (SLI1) and 19q (SLI2). This was followed by a replication of both regions in an additional 86 families. Both these studies applied linkage methods to one phenotypic trait at a time. However, investigations have suggested that simultaneous analysis of several traits may offer more power. The current study therefore applied a multivariate variance-components approach to the SLI Consortium dataset using additional phenotypic data. A multivariate genome scan was completed and supported the importance of the SLI1 and SLI2 loci, whilst highlighting a possible novel QTL on chromosome 10. Further investigation implied that the effect of SLI1 on non-word repetition was equally as strong on reading and spelling phenotypes. In contrast, SLI2 appeared to have influences on a selection of expressive and receptive language phenotypes in addition to non-word repetition, but did not show linkage to literacy phenotypes.

Keywords: Non-word repetition, CELF-R, multivariate, variance components, SLI, Mx

\section{Introduction}

During development it is expected that children will learn their native language relatively quickly and easily and, by the age of four, be able to construct long sentences with a vocabulary of up to 5000 words (O'Grady 2005). There is a proportion of children, however, who appear to have difficulty in this development. In many cases this is caused by other medical or developmental problems, such as autism, deafness or cleft lip and palate. Nevertheless, $3 \%-7 \%$ of preschool children have a language impairment but appear to have no known medical problems that can explain the language delay (Tomblin et al. 1997; Law et al. 1998). Furthermore, they have normal non-verbal intelligence and have had adequate

* Correspondence to: Anthony P. Monaco Wellcome Trust Centre for Human Genetics, University of Oxford, Oxford, OX3 7BN, United Kingdom. Tel: +44 (0) 1865287 502/503. Fax: +44 (0) 1865287 650; E-mail: Anthony.Monaco@well.ox.ac.uk

${ }^{1}$ For a list of members please see appendix. opportunity to improve their language skills. This disorder is defined as Specific Language Impairment (SLI [MIM 606711]).

Studies published in the 1980s and 1990s consistently demonstrated familial aggregation in cases of language impairment (Bishop \& Edmundson, 1986; Neils \& Aram, 1986; Tallal et al. 1989; Stromswold, 1998). Subsequent twin studies indicated an increase in concordance rates of $\mathrm{MZ}$ twin pairs over that of $\mathrm{DZ}$ twins, suggesting that much of the familial aggregation can be attributed to genetic influences (Lewis \& Thompson, 1992; Bishop et al. 1995; Tomblin \& Buckwalter, 1998; Viding et al. 2004). Furthermore, heritability of language ability has been suggested to increase as a function of the severity of language impairment. In their twin study, Viding et al. (2004) reported a group heritability of $41 \%$ when probands were defined as having a composite language score 1.5SDs below the mean. This figure rose to $72 \%$ when a more severe cut-off of 3 SDs was applied (Viding et al. 2004). 
In recent years two groups have published genome scans specifically looking for linkage to SLI. Bartlett et al. (2002) studied 84 individuals selected from five extended Canadian pedigrees, originally ascertained for a schizophrenia study. Each family included at least two individuals with a spoken language measure $>1 \mathrm{SD}$ below that expected for their age. All individuals with a performance IQ below 80, hearing outside the normal limits, motor impairments or oral structural deviations, or a diagnosis of autism, schizophrenia, psychoses or neurological disorders, were excluded. Three dependent categorical phenotypes were analysed using dominant and recessive models (reading impaired, language impaired and clinically impaired). Results showed linkage at $13 \mathrm{q} 21$ using the recessive reading model (maximum LOD 3.92) (SLI3 [MIM607134]), and at 2p22 using the recessive language model (maximum LOD 2.86). A replication of the $S L I 3$ locus was achieved two years later using an independent sample of 22 American families, both nuclear and extended. These families were clinically referred and again contained at least two affected individuals. The assessment battery and linkage approach were identical to those used previously, but in this replication study they only typed markers on chromosomes 2, 7 and 13. Linkage was again observed on chromosome $13 \mathrm{q}$ under a recessive model of reading impairment, yielding a maximum LOD score of 2.62 (Bartlett et al. 2004).

The SLI Consortium (2002) collected a sample of 98 families, all containing at least one proband with language skills $>1.5 \mathrm{SD}$ below the mean for their chronological age. In contrast to Bartlett et al. (2002, 2004), they directly utilised quantitative measures of language ability. Three phenotypes - non-word repetition (NWR), and the Clinical Evaluation of Language Fundamentals - Revised (CELF-R) expressive and receptive language scales - were analysed using Haseman-Elston (HE) and variance components (VC) linkage approaches. Two loci exceeded suggestive levels for genome-wide significance. A QTL on chromosome 16q (SLI1 [MIM606711]) showed linkage using the non-word repetition phenotype (LOD 3.55). The second QTL, on chromosome 19q, provided evidence of linkage using the expressive language score (LOD 3.55) (SLI2 [MIM606712]). Replication of both of these loci was achieved using a second cohort of 86 families with

(C) 2007 The Authors

Journal compilation (C 2007 University College London identical selection criteria and assessment battery (The SLI Consortium, 2004). However, within this sample the non-word repetition phenotype showed linkage to both regions (chromosome 16 maximum LOD 2.84, chromosome 19 maximum LOD 2.31), whereas the expressive language score showed no linkage to either chromosome. By combining the two waves of data the LOD score on chromosome 16 increased to 7.46 , whilst that on chromosome 19 decreased to 1.40 . This was attributed to the fact that the separate waves were primarily linked to two different phenotypes in this region. In their replication study the SLI Consortium also demonstrated suggestive levels of linkage between a measure of spelling ability and the SLI1 region (maximum LOD 2.67).

In addition to these full genome screens, some investigators have performed linkage analyses in specific chromosomal regions which have previously been linked to disorders associated with SLI. These studies have all been performed using families affected by speech-sound disorder (SSD), characterised by developmentally inappropriate errors in speech production and considered to be a phonological subtype of SLI (Stein et al. 2004, 2006; Smith et al. 2005). The first study of SSD (Stein et al. 2004) focussed upon a region of chromosome 3 previously implicated in dyslexia (DYX5 [MIM606896]). Probands and siblings were assessed on a battery of tests covering phonological memory, articulation, vocabulary (expressive and receptive), reading and reading, comprehension abilities. These data were then used to derive factor scores for articulation, phonological memory and vocabulary, which were then analysed for linkage by applying a model-free approach. The strongest significant linkage was found using the phonological factor score $\left(P=5.6 \times 10^{-5}\right)$ [MIM608445]. Evidence for linkage was also found using the reading scores $(P<0.006)$. The same set of families were also used in an investigation of a region of chromosome $15 q$ which is deleted in Prader-Willi and Angelman Syndromes and has been linked to both autism (AUTS4 [MIM209850]) and dyslexia (DYX1 [MIM127700]) (Stein et al. 2006). The factor scores were not employed in this study, but instead an affected sib-pair analysis was performed using a binary affection status. Linkage was found within a Caucasian subgroup of families $(P=0.007)$, which strengthened when quantitative measures of oral-motor 
function, articulation and phonological memory were included as covariates within a conditional logistic model $(P=0.004)$. A second group also found linkage to chromosome $15 \mathrm{q}$ in a cohort of families affected by SSD. Smith et al. (2005) specifically investigated loci previously implicated in dyslexia on chromosomes $1 \mathrm{p}$ (DYX8 - [MIM608995]), 6p (DYX3 - [MIM604254]) and 15q (DYX1 - [MIM127700]) within 65 families affected by SSD. They employed both Haseman-Elston and DeFries-Fulker methodology to analyse a qualitative diagnosis of affection status alongside six quantitative measures of spoken language ability at these loci. They reported significant linkage to the affection status on chromosome $6(P=0.0006)$, and to measures of nonword repetition and articulation on chromosome $15 \mathrm{q}$. It therefore appears that the results of studies of families affected by SSD overlap with those of dyslexia in a way that investigations of SLI do not. This reflects the aetiological complexities documented by behavioural studies (Lewis et al. 2000; Raitano et al. 2004) and may, in part, be attributable to the fact that a diagnosis of dyslexia or SSD relies heavily upon the presence of phonological deficits, whereas SLI may involve impairments in alternative language domains (e.g. morphology, syntax, semantics).

The studies described above conveniently illustrate the issues which surround both the design of and the interpretation of results from, investigations into complex genetic disorders such as SLI. SLI is phenotypically heterogeneous, making it difficult to obtain a meaningful and consistent affection status, and often resulting in a lack of agreement in the methodologies applied between studies. In addition, there are substantial behavioural and aetiological overlaps with other developmental disorders such as SSD, dyslexia, attention deficit disorder and pervasive developmental disorders (Beitchman \& Inglis, 1991; Bishop \& Snowling, 2004), indicating the probable existence of pleiotropic genetic effects. Moreover, the direct comparison of results between studies is often further complicated by the application of alternative linkage methods, and the way in which different researchers report the significance levels of the loci identified (e.g. $p$-values, LOD scores, $Z$-scores etc). The extent of linkage found by any given study, therefore, not only reflects the genetic effects operating within that sample, but is also affected by factors such as the sensi- tivity and distribution of the psychometric and linkage tests used, and the ascertainment and size of the samples collected (Fisher \& DeFries, 2002).

Researchers are increasingly turning to multivariate techniques for the analysis of correlated traits. Such techniques simultaneously consider quantitative measures within a linkage model and account for the phenotypic correlations between traits (Marlow et al. 2003; Wang \& Elston, 2007). They can therefore increase the accuracy of linkage estimates and the power to detect QTLs (Boomsma \& Dolan, 1998), especially when there is a large genetic correlation between the traits (Schmitz et al. 1998). In addition, replacing a set of univariate analyses with a single multivariate analysis removes the need to correct for the multiple testing of correlated traits that can result in over-conservative results. Furthermore, multivariate linkage analysis may be used to dissect genes with pleiotropic effects, as is expected to be the case for developmental disorders such as SLI. The current study therefore applies the multivariate method of Vogler et al. (1997) and Marlow et al. (2003) to the SLI Consortium (SLIC) data. This has allowed us simultaneously to investigate the genetic effects upon each of the measures collected by the previous SLIC studies, and to clarify the common and specific effects at the SLI1 and SLI2 loci identified by these investigations.

\section{Subjects and Methods}

\section{Subjects}

In total 840 individuals were recruited from 184 families by four separate centres - the Newcomen Centre at Guy's Hospital, London; the Cambridge Language and Speech Project (CLASP); the Child Life and Health Department at the University of Edinburgh; and the Department of Child Health at the University of Aberdeen. All families were selected via a single proband who, either currently or in the past, had language skills $\geq 1.5$ SD below the normative mean for their chronological age on the receptive and/or expressive scales of the CELF-R battery (Semel et al. 1992). Any proband or sibling found to have a performance IQ of below 80 was excluded from the sample. Full details of all cohort structures and ascertainment criteria can be found in 
previous publications from The SLI Consortium (2002, 2004).

The model applied to the data was only defined for full sibships of magnitude 2-4 with adequate genetic data. After excluding any half-siblings to each proband six families (22 individuals) only contained one remaining offspring (the proband) and therefore were omitted from the analysis. Since the Aberdeen sample is small we combined it with the Guy's sample; this is consistent with the approach used for previous analyses (The SLI Consortium, 2004).

The entire cohort can be considered in three separate ways: in terms of the site at which the proband was identified (i.e. Guy's and Aberdeen, Cambridge, Edinburgh); in terms of the nature of the site at which they were identified (i.e. Clinical selection - Edinburgh, Guy's and Aberdeen, or epidemiological selection - Cambridge); or in terms of chronologically defined 'waves' (i.e. those samples collected from Guy's and Cambridge prior to 2002 and used for the SLIC whole genome screen (The SLI Consortium 2002) - wave 1, and those subsequently collected by all four of the sites and used for the SLIC replication study of chromosomes 16 and 19 (The SLI Consortium 2004) - wave 2).

\section{Phenotypic Measures}

Expressive and receptive language abilities were assessed separately, using the language test battery CELF-R (Semel et al. 1992), by obtaining a score from three of the following tests, depending on the age and language ability of the child. The expressive language subtests included formulating sentences (FSENT), recalling sentences (RSENT), sentence assembly (SA) and word structure (WDST). The receptive language subtests were oral directions (OD), word classes (WC), semantic relationships (SREL), sentence structure (SST) and linguistic concepts (LCON). These tests can be analysed separately or used to create two cumulative scores - one expressive (ELS) and the other receptive (RLS). Due to small sample sizes, the measures for the under 8's only, WDST, SST and LCON, were excluded from this study.

Literacy was tested using the WORD assessment (Rust et al. 1993), designed for children of ages 6 to 16. WORD consists of three components, each measuring a separate aspect of literacy: single-word reading
(BRSS), single-word spelling (SPSS), and reading comprehension (RCSS). Due to logistical constraints the WORD tests were only performed for the Guy's and Cambridge samples.

Non-word repetition (NWR) requires the child to repeat nonsense words after hearing them on a cassette player (Gathercole et al. 1994). There is evidence to suggest that individuals with current and resolved language difficulties are impaired on this test (Bishop et al. 1996).

In addition to the language measures, performance IQ (PIQ) was assessed from the Wechsler Intelligence Scale for Children - III (WISC-III) (Wechsler, 1992).

Thus, the SLIC samples had a total of 13 tests available for multivariate analyses (ELS, RLS, FSENT, RSENT, SA, OD, WC, SREL, BRSS, SPSS, RCSS, NWR and PIQ).

\section{Statistical Analyses}

All traits were standardised within each sample and checked to be normally distributed. Traits FSENT, RSENT and SA were transformed before standardising using square root (FSENT and RSENT) and natural logarithm functions (SA) so that the data were normal. Correlations were calculated using the SPSS package.

In order to determine whether the underlying parameters of each trait are similar for each sample, heterogeneity tests were also performed. For each trait a chi-squared $\left(\chi^{2}\right)$ statistic was calculated using the loglikelihoods of the full model, using separate parameter sets for each sample, and its submodel where the familial effects were equal across samples (see Supplementary data, Table 1). In the wave 1 and 2 combined sample significant differences were found for the parameter estimates for the CELF subtest scores in the Cambridge sample compared to the other three clinically derived samples (see Supplementary data, Table 1). We therefore decided to fit separate parameters for the background familial and unique environment components across samples. This resulted in two sets of parameters in the multivariate model - one for Cambridge (the epidemiological sample) and another for Aberdeen, Guy's and Edinburgh (the combined clinical sample). Although the wave 1 data did not show significant heterogeneity between the Cambridge and Guy's samples, the 
Table 1a Significant correlations at the 1\% level for waves 1 and 2 combined

\begin{tabular}{|c|c|c|c|c|c|c|c|c|c|c|c|c|}
\hline & Sex & PIQ & OD & WC & SREL & FSENT & RSENT & SA & BRSS & SPSS & RCSS & NWR \\
\hline Sex & 1 & & & & & & & & & & & \\
\hline PIQ & & 1 & & & & & & & & & & \\
\hline OD & & 0.467 & 1 & & & & & & & & & \\
\hline WC & & 0.401 & 0.594 & 1 & & & & & & & & \\
\hline SREL & & 0.444 & 0.633 & 0.652 & 1 & & & & & & & \\
\hline FSENT & 0.103 & 0.418 & 0.541 & 0.515 & 0.521 & 1 & & & & & & \\
\hline RSENT & & 0.360 & 0.569 & 0.686 & 0.643 & 0.574 & 1 & & & & & \\
\hline SA & 0.122 & 0.398 & 0.527 & 0.559 & 0.634 & 0.570 & 0.546 & 1 & & & & \\
\hline BRSS & & 0.453 & 0.561 & 0.605 & 0.662 & 0.502 & 0.567 & 0.639 & 1 & & & \\
\hline SPSS & 0.138 & 0.401 & 0.507 & 0.541 & 0.626 & 0.460 & 0.519 & 0.632 & 0.843 & 1 & & \\
\hline RCSS & 0.130 & 0.529 & 0.564 & 0.620 & 0.656 & 0.513 & 0.552 & 0.617 & 0.765 & 0.690 & 1 & \\
\hline NWR & & 0.248 & 0.433 & 0.431 & 0.383 & 0.413 & 0.526 & 0.386 & 0.502 & 0.453 & 0.439 & 1 \\
\hline
\end{tabular}

PIQ, Wechsler Performance (non-verbal) IQ; OD, CELF Oral Directions (subtest of the Receptive Language Score (RLS)); WC, CELF Word Class (subtest of the Receptive Language Score (RLS)); SREL, CELF Semantic Relations (subtest of the Receptive Language Score (RLS)); FSENT, CELF Formulating Sentences (subtest of the Expressive Language Score (ELS)); RSENT, CELF Recalling Sentences (subtest of the Expressive Language Score (ELS)); SA, CELF Sentence Assembly (subtest of the Expressive Language Score (ELS)); BRSS, WORD Single Word Reading Score; SPSS, WORD Single Word Spelling Score; RCSS, WORD Reading Comprehension Score; NWR, Non-word repetition.

Table 1b Wave one significant correlations at the 1\% level

\begin{tabular}{lllll}
\hline & ELS & RLS & NWR & PIQ \\
\hline ELS & 1 & & & \\
RLS & 0.766 & 1 & & \\
NWR & 0.544 & 0.494 & 1 & \\
PIQ & 0.451 & 0.490 & 0.187 & 1 \\
\hline
\end{tabular}

ELS, Expressive Language Score; RLS, Receptive Language Score; NWR, Non-word repetition; PIQ, Wechsler Performance (non-verbal) IQ.

above method was also applied to the multivariate model in wave 1 in order to gain an improved fit to the data.

Since the families were selected through a severely affected proband, ascertainment-corrected estimates of familiality were calculated using SOLAR (Almasy \& Blangero, 1998). (see Supplementary data, Table 1). For the combined wave 1 and 2 sample the variance components (VC) approach resulted in familiality estimates close to zero for the clinical sample (see Supplementary data, Table 1). Although it is disquieting to see familiality values close to zero, it is not an uncommon phenomenon in populations where the variance of the trait is restricted by a selection procedure (Amos \& deAndrade, 2001). For this sample an increase in power with regards to familiality estimates may be obtained by the use of DeFries-Fulker methodology (DeFries
\& Fulker, 1985). In contrast to variance components techniques, which derive familiality estimates via the partition of the variance, DeFries-Fulker analyses use multiple regression to estimate group heritability of extreme scores. This method is known to be more robust to selected samples. By using the DeFries-Fulker method we obtained significant clinical familiality estimates for all but two traits (11.9\% for WC and $12.1 \%$ for SA, all other familiality values between $21.8 \%$ and $55.0 \%$ - data not shown). However, as a precautionary measure the clinical QTL parameters for each of the CELF subtests were omitted from the multivariate linkage analysis for the wave 1 and 2 combined sample on chromosomes 16 and 19.

\section{Multivariate Analysis}

The multivariate analysis used in this study was developed by Marlow et al. (2003) where the familial background and unique environmental components were modelled using the saturated Cholesky framework. The QTL effects were considered using a single QTL factor, with effect $\mathrm{q}_{k}$ on trait $k$. The phenotypic variance due to the QTL is therefore defined as $\mathrm{q}_{k}^{2}$ and the QTL covariance between trait $\mathrm{k}$ and trait $\mathrm{k}^{\prime}$ is estimated as $\mathrm{q}_{k} \mathrm{q}_{k^{\prime}}$. The model was incorporated into the Mx package (Neale et al. 2002) using multipoint IBD values estimated by 
Genehunter (Kruglyak et al. 1996). In Mx, the closest fit to the data for each point on the chromosome was obtained by maximum likelihood methods.

Thus the multivariate analyses proceeded as follows. In order to minimise the degrees of freedom, a full genome screen was completed for the wave 1 sample using a multivariate model including just three traits (ELS, RLS and NWR), with QTL parameters equated across all samples. Any regions exceeding a $P=0.01$ threshold of linkage were then further investigated using the minimal three trait model in the larger waves 1 and 2 combined cohort. In order to determine which traits contributed to each QTL, we performed a 'trait dropping analysis' in which submodels were fitted at each peak for each trait $\mathrm{k}$ and sample 1 , restricting the QTL parameter $\mathrm{q}_{k}$, for trait $\mathrm{k}$, to zero. The submodel was then compared to the full model using a $\chi^{2}$ test with one degree of freedom. If no significant difference was detected at the $5 \%$ level, we excluded the trait from the model at the QTL. This was calculated by a $\chi^{2}$ test on $\mathrm{m}$ degrees of freedom, where $m$ is the number of parameters dropped when excluding the QTL effect. It has been shown that the distribution of $\chi^{2}$ statistics for univariate variance components comprises a 50:50 mixture of $\chi^{2}$ distributions of 1 degree of freedom and point mass 0 (Self $\&$ Liang, 1987). The underlying $\chi^{2}$ distribution for multivariate variance components is thought to be more complex (Amos et al. 2001; Marlow et al. 2003), but due to lack of research into this problem the more conservative $\chi^{2}$ test on $m$ degrees of freedom was used instead. Following these parameter reduction procedures, we looked for further parsimony by equating a subset of the significant QTL parameters. By setting $q_{k}=q_{k^{\prime}}$ for traits $k$ and $k$ ' we were able to test whether the two traits have similar QTL effects. In line with previous publications on linkage analysis (The SLI Consortium 2002, 2004), we present pointwise $p$-values.

In addition, chromosomes 16 and 19 were investigated in full detail using all available traits within the wave 1 and 2 combined sample. This was again achieved using a two step modelling process. The initial multivariate model contained 7 parameters - the 6 CELF subtests (FSENT, RSENT, SA, OD, WC, SREL) for the Cambridge cohort only, and the NWR parameter equated across all samples. This was followed by a full model which included the significant traits from the first stage, in combination with the three WORD tests and PIQ (if significant). The final model in these regions therefore contained a maximum of eleven traits - the 6 CELF subtests (FSENT, RSENT, SA, OD, WC and SREL), non-word repetition (NWR), performance IQ (PIQ) and the three WORD measures (BRSS, SPSS and RCSS).

Finally, since deviations from multivariate normality and known difficulties with the $\chi^{2}$ distribution of multivariate variance components models can cause unpredictable variations between nominal and true $p$-values, we conducted permutations of the data to obtain empirical p-values. At each identified QTL the estimated multipoint IBD values were permuted between sib-pairs to obtain 1000 datasets, while maintaining the pedigree structure and phenotype data. The most parsimonious model (i.e. a multivariate model containing only those QTL parameters which were found to be significant in the trait dropping analysis) at each QTL was then applied to the dataset, in order to obtain the empirical p-value distribution. Results implied that all models tested were overly conservative, leading to reduced type I errors but increased type II errors ( see Supplementary data, Table 2).

\section{Results}

\section{Descriptive Statistics}

Descriptive statistics of the wave 1 , and waves 1 and 2 combined, samples can be found in Supplementary Table 3. As may be expected, both the Edinburgh and the Guy's and Aberdeen samples were found to have lower mean scores than the Cambridge sample when considering either proband or sibling scores. This is due to the fact that the clinical samples have persistent language problems severe enough to require special schooling, and are therefore not representative of the total population in the community. The one exception was for sentence assembly (SA) where the Cambridge means (7.4 proband, 8.2 sibling) were lower than both the Edinburgh (7.4 proband, 8.7 sibling) and Guy's and Aberdeen (7.7 proband, 9.7 sibling) groups (see Supplementary Table 3). The lowest score in each sample was formulating sentences, followed by recalling sentences, within the clinical samples (see Supplementary Table 3). 
Table 2 Testing the contribution of each trait to the multivariate linkage on chromosomes 1, 4, 5, 7, 10,16 and 19 for wave 1.

\begin{tabular}{llllllll}
\hline Trait & Chr 1 $(300 \mathrm{cM})^{\mathrm{a}}$ & Chr $4(58 \mathrm{cM})^{\mathrm{a}}$ & $\mathrm{Chr} 5(71 \mathrm{cM})^{\mathrm{a}}$ & $\mathrm{Chr} 7(2 \mathrm{cM})^{\mathrm{a}}$ & $\mathrm{Chr} 10(163 \mathrm{cM})^{\mathrm{a}}$ & $\mathrm{Chr}^{16}(115 \mathrm{cM})^{\mathrm{a}}$ & $\mathrm{Chr} 19(77 \mathrm{cM})^{\mathrm{a}}$ \\
\hline ELS & $\mathbf{0 . 0 0 1 6}$ & $\mathbf{0 . 0 0 0 5}$ & $\mathbf{0 . 0 1 1 0}$ & $\mathbf{0 . 0 0 0 1}$ & 0.5639 & $\mathbf{0 . 0 0 0 6}$ & $\mathbf{0 . 0 0 0 9}$ \\
RLS & 0.7530 & $\mathbf{0 . 0 0 9 9}$ & 0.4260 & $\mathbf{0 . 0 3 5 0}$ & $\mathbf{0 . 0 0 9 8}$ & $\mathbf{0 . 0 0 1 0}$ & $\mathbf{0 . 0 1 6 4}$ \\
NWR & 0.4045 & 0.1413 & $\mathbf{0 . 0 0 8 1}$ & 0.5371 & $\mathbf{0 . 0 0 2 5}$ & $\mathbf{0 . 0 0 0 2}$ & 0.0727 \\
\hline
\end{tabular}

${ }^{a}$ The position indicates the peak of the linkage. This is the point at which we tested whether the traits could be dropped from the multivariate model.

Nominal $p$-values exceeding the $5 \%$ level, shown in bold, indicated that the trait cannot be dropped from the multivariate model at the QTL and therefore contributed to the linkage. ELS, Expressive Language Score; RLS, Receptive Language Score; NWR, Non-word repetition.

Table 3 Testing the contribution of each trait to the multivariate linkages on chromosomes 16 and 19 for waves 1 and 2 combined, using the six CELF traits and NWR

\begin{tabular}{llll}
\hline Trait & Chr 16 $(151 \mathrm{cM})$ & Chr 19 $(106 \mathrm{cM})$ & Chr 19 $(174 \mathrm{cM})$ \\
\hline OD $^{\mathrm{a}}$ & $\mathbf{0 . 0 4 1 1}$ & $\mathbf{0 . 0 0 1 4}$ & 0.3455 \\
WC $^{\mathrm{a}}$ & 0.2476 & $\mathbf{0 . 0 0 7 8}$ & 0.2321 \\
SREL $^{\mathrm{a}}$ & 0.3554 & $\mathbf{0 . 0 1 8 2}$ & $\mathbf{0 . 0 0 5 9}$ \\
FSENT $^{\mathrm{a}}$ & 0.8002 & $\mathbf{0 . 0 0 1 1}$ & 0.3292 \\
RSENT $^{\mathrm{a}}$ & 0.1566 & $\mathbf{0 . 0 0 2 2}$ & $\mathbf{0 . 0 0 1 7}$ \\
SA $^{\mathrm{a}}$ & 0.8648 & $\mathbf{0 . 0 0 0 9}$ & 0.0921 \\
NWR & $\mathbf{0 . 0 0 2 6}$ & $\mathbf{0 . 0 6 2 6}$ & $\mathbf{0 . 0 2 0 8}$ \\
PIQ $^{\mathrm{b}}$ & 0.5919 & 0.0655 & 0.9807 \\
\hline
\end{tabular}

${ }^{a}$ Cambridge sample only.

${ }^{\mathrm{b}}$ The significance of PIQ was tested separately, using the full multivariate model for each chromosome. Note that there are two QTL parameters for PIQ across samples, therefore this test was performed on two degrees of freedom.

Nominal $p$-values exceeding the $5 \%$ level, shown in bold, indicated that the trait could not be dropped from the multivariate model at the QTL and therefore contributed to the linkage. OD, CELF Oral Directions (subtest of the Receptive Language Score (RLS)); WC, CELF Word Class (subtest of the Receptive Language Score (RLS)); SREL, CELF Semantic Relations (subtest of the Receptive Language Score (RLS)); FSENT, CELF Formulating Sentences (subtest of the Expressive Language Score (ELS)); RSENT, CELF Recalling Sentences (subtest of the Expressive Language Score (ELS)); SA, CELF Sentence Assembly (subtest of the Expressive Language Score (ELS)); NWR, Non-word repetition; PIQ, Wechsler Performance (non-verbal) IQ.

Interestingly, although the Edinburgh probands were selected on the basis of their receptive language abilities, they also achieved low scores for the CELF subtests which measure expressive language (FSENT, RSENT and SA). This supports the hypothesis that individuals with a receptive language impairment usually have respectively poor expressive language abilities (Bishop et al. 1995). Further evidence for this theory is provided by the significant correlations between the CELF expressive (FSENT, RSENT and SA) and receptive (OD, WC and SREL) subtests scores $(0.52-0.69-$ Table $1 \mathrm{a})$.

Correlations between the CELF (FSENT, RSENT, SA, OD, WC and SREL) and WORD (BRSS, SPSS and RCSS) measures were all high, between 0.460 (FSENT, SPSS) and 0.843 (BRSS, SPSS), with the strongest correlations between the WORD tests (Table 1a). In general, correlations involving NWR were slightly lower, between 0.383 (SREL) and 0.544 (ELS wave 1 only) (Tables $1 \mathrm{a}$ and $1 \mathrm{~b})$. Correlations with performance IQ ranged from 0.187 (NWR, wave 1) to 0.526 (RSENT, wave 1 and 2). No sex differences were evident (Table 1a) and adding the sex variable as a covariate made no difference to the multivariate linkage analysis (results not shown). The covariate was therefore excluded from the linkage model.

\section{Wave 1 Genome Scan}

The results of the multivariate wave 1 genome scan, using the ELS, RLS and NWR traits, are presented in Figure 1. Evidence for linkage with a nominal p-value of less than 0.01 was found on chromosomes 1, 4, 5, 7, 10,16 and 19 (p-values $0.0045,0.0048,0.0039,0.0002$, $0.0051,0.0032$ and 0.0073 respectively).

By testing which QTL effects were significant at each peak, we were able to reduce the number of QTL parameters for each model to find the best fit to the data (Table 2). This approach decreases the degrees of freedom, and also gives some insight into which traits correlate with the QTL and how the QTL parameters relate to each other. For example, on chromosome 1 it appeared that a single trait (ELS) contributed to the locus (Table 2). In contrast, all the other putative QTLs 


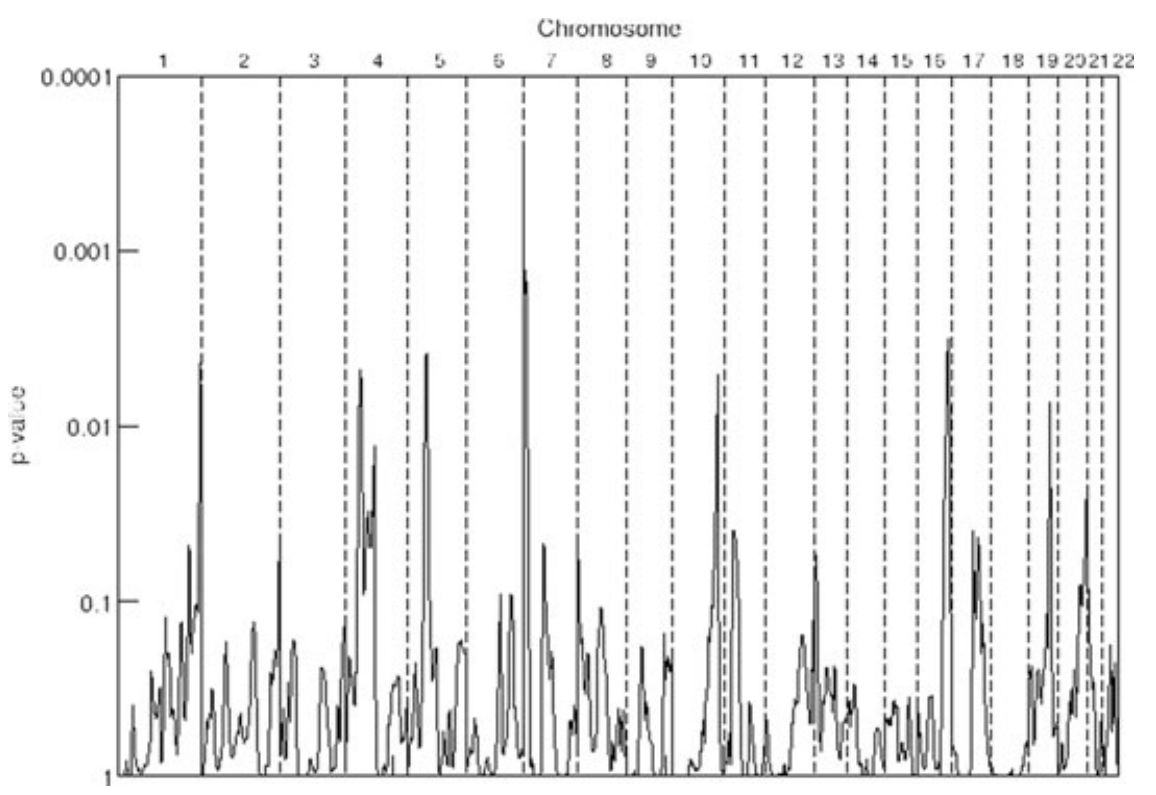

Figure 1 Complete multivariate genome screen of ELS, RLS and NWR for the wave 1 sample.

appeared to be caused by various combinations of the three traits under study. On chromosomes 4, 7 and 19 both ELS and RLS were significant components of the multivariate model (Table 2). However, whilst on chromosomes 4 and 19 it appeared that these two traits contribute equally to the QTL, on chromosome 7 the two parameters behaved differently from each other. Similarly, on chromosomes 5 and 10, two traits contributed to the QTL (ELS and NWR on chromosome 5 and RLS and NWR on chromosome 10 - Table 2), each with significantly different effects. Although all three traits were significant on chromosome 16 (Table 2), NWR had a stronger QTL effect than ELS and RLS, which did not have significantly different QTL effects. The QTL effects at each peak under these 'most-parsimonious' models remained significant when assessed by permutation, and in most cases the permutation-based pointwise $p$-values obtained were less than the nominal $\mathrm{p}$-values (Supplementary Table 2).

We then went on to investigate whether any of the loci identified by the full genome screen could be replicated within the larger dataset, which incorporated the wave 1 and 2 samples. Of the seven loci identified by the genome screen, only chromosome 16 remained significant $(P<0.01)$ within the wave 1 and 2 sample $(P=0.00855-$ Figure 2$)$.

\section{Waves 1 and 2 combined - chromosome 16.}

The initial chromosome 16 follow-up multivariate model used genotypic data from across chromosome 16 and phenotypic data from seven language-related measures - the NWR measure and the 6 CELF subtests (FSENT, RSENT, SA, OD, WC and SREL). In contrast to the above multivariate investigations this approach considered each of the individual CELF subtests, rather than the cumulative expressive and receptive language scores, and produced the multivariate linkage results presented in Figure 3a. The increased test battery, however, conferred an increase in the number of degrees of freedom $(n=7)$, which may account for the poor significance (nominal $p$-value $>0.1$ ) of the multivariate results, especially since additional investigations revealed that only two QTL parameters were significant across this region (OD Cam and NWR Table 3). The model was therefore adjusted, restricting all non-significant QTL parameters to zero, reducing the degrees of freedom to 2 ('mv adjusted', Figure 3a), and yielding a nominal $p$-value of 0.026 at the peak of linkage.

The final, full multivariate model on chromosome 16 therefore contained five parameters, the significant traits from the follow-up model (OD_Cam and NWR) and an additional three WORD measures (BRSS, SPSS and 

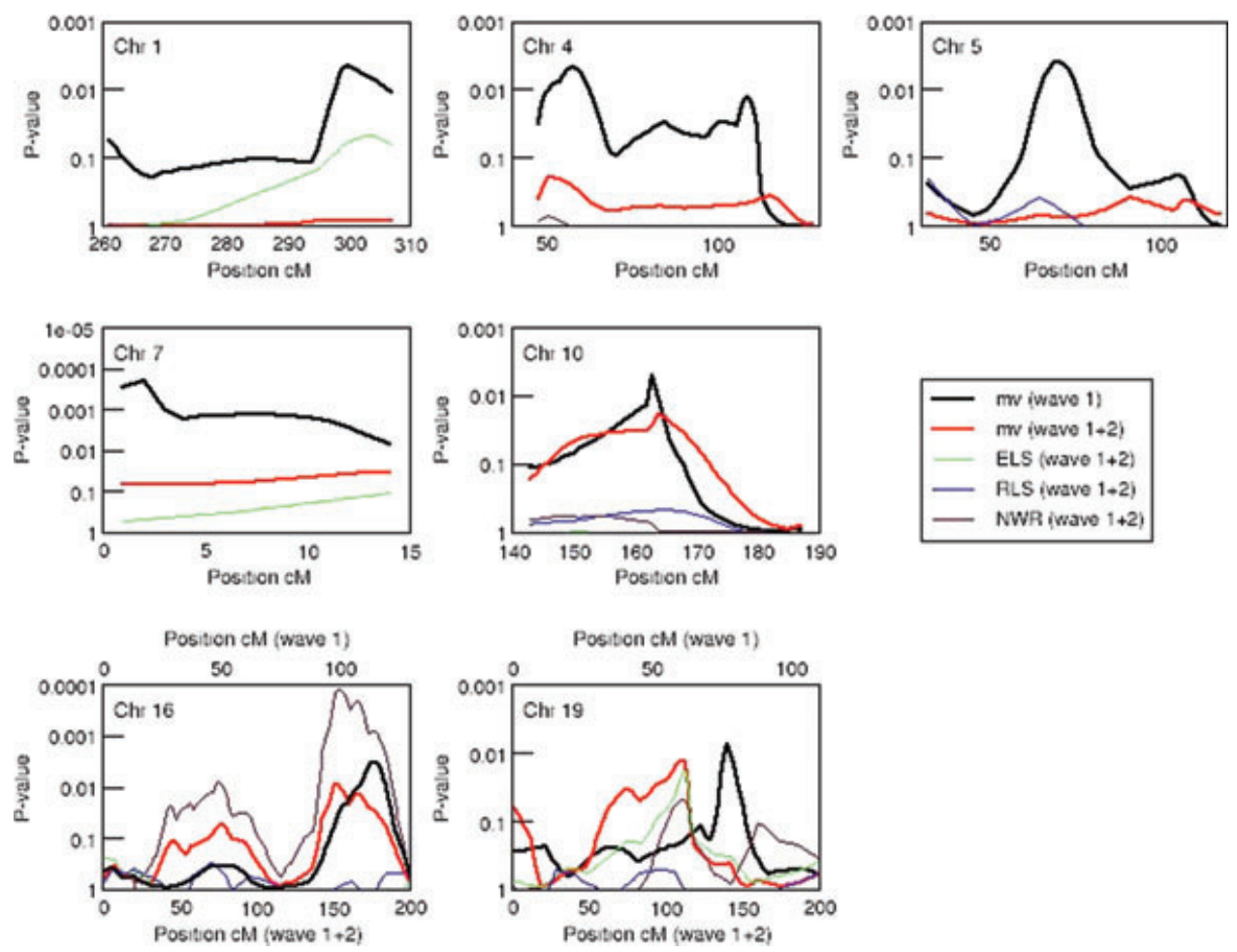

Figure 2 Multivariate and univariate analysis of the three language traits for chromosomes 1, 4, 5, 7, 10, 16 and 19 for the wave 1 and combined wave 1 and 2 samples. Mv (wave 1) and $\mathrm{mv}$ (wave $1+2$ ) represent the nominal p-values for the multivariate peaks for the three parameter model in wave 1 and the wave $1 \& 2$ combined samples respectively. All other traces represent the nominal $\mathrm{p}$-values for the univariate analysis of the respective trait (ELS - CELF expressive language score, RLS - CELF receptive language score, NWR - non-word repetition).

RCSS). Again, the increase in degrees of freedom resulted in a decrease in linkage significance, which was improved upon by dropping non-significant QTL parameters (RCSS) ('mv' vs 'mv adjusted' Figure 3b, nominal $p$-value $=0.0089$ ). The two analyses indicated that the QTL effect of NWR was much stronger than that of OD, but equally as strong as the reading and spelling tests from the WORD test battery.

By incorporating the PIQ measure into our analyses as an additional phenotype, we determined that performance IQ did not contribute to the QTL within this region (Table 3).

\section{Waves 1 and 2 combined - chromosome 19}

The follow-up analysis of chromosome 19 used the same initial seven traits as used on chromosome 16 (the NWR measure and the 6 CELF subtests (FSENT, RSENT, SA, OD, WC and SREL)). Within this model we found striking significant linkage to the distal end of this re- gion with a maximal nominal p-value of $0.0008(174 \mathrm{cM}$ - Figure 3c). This region of linkage is distal to that identified by the multivariate genome screen, and is towards the telomeric end of the wider peak originally described in the univariate linkage analyses (The SLI Consortium, 2002, 2004). The dropping of QTL parameters indicated that this linkage could be attributed to the two CELF subtests SREL (receptive) and RSENT (expressive), and to a lesser extent NWR. Across the original proximal region of interest all of the 6 CELF subtest QTL parameters (OD, WC, SREL, FSENT, RSENT and $\mathrm{SA}$ ) were significant within the Cambridge cohort (106cM - Table 3). Across this region we found that equating the significant QTL parameters did not make a significant difference to the model, indicating that no single trait has a larger influence on the linkage compared to the other measures.

The incorporation of the three WORD reading tests and a measure of PIQ into the multivariate model indicated that none of these measures contributed 


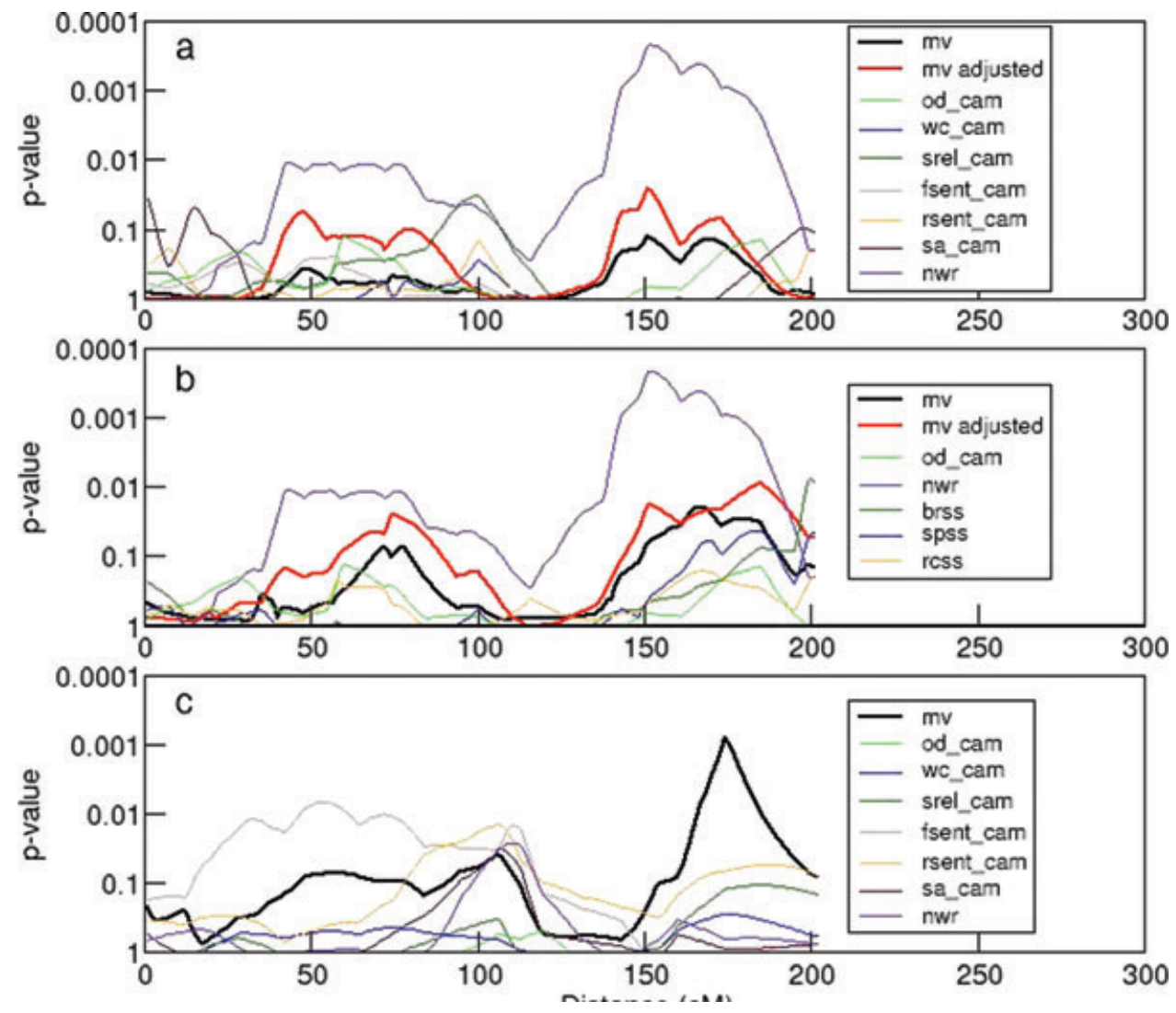

Figure 3 Multivariate and univariate analysis for the combined wave 1 and 2 sample for chromosomes 16 and 19. (a) The chromosome 16 multivariate model including all six CELF subtests (OD, WC, SREL, FSENT, RSENT, SA (Cambridge only)) and non-word repetition (NWR). (b) The chromosome 16 multivariate model including any traits from analysis A with $p<0.05$ (OD and NWR), together with the three WORD reading tests (BRSS, SPSS and RCSS). (c) The chromosome 19 multivariate model including all six CELF subtests (OD, WC, SREL, FSENT, RSENT, SA (Cambridge only)) and non-word repetition (NWR). Note that the three reading measures were found to be non-significant in this region and are therefore not shown here. 'mv' trace represents multivariate model including all parameters. 'mv_adjusted' trace represents the multivariate model of best fit. In these models all QTL effects with $P<0.05$ have been removed. All p-values are nominal. All other traces represent the univariate analysis of the respective trait (OD - CELF oral directions, WC - CELF word classes, SREL - CELF semantic relations, RSENT - CELF recalling sentences, SA - CELF sentence assembly, NWR - non-word repetition, BRSS - WORD single word reading, SPSS WORD single word spelling, RCSS - WORD reading comprehension). All p-values are nominal. The suffix '_cam' indicates that these QTL parameters were only significant in the Cambridge (epidemiological) sample.

significantly to the multivariate linkage at either region of chromosome 19.

\section{Discussion}

This report describes a genome-wide multivariate analysis of three language-related measures (CELF composite scores for expressive and receptive language (ELS and RLS, respectively) and Non-Word Repetition (NWR)) in a sample of 98 families affected by language impair- ment. This genome scan enabled the identification of a novel putative QTL on chromosome 10 and provided further support for two loci previously identified on chromosomes 16q and 19q (SLI1 and SLI2, respectively - The SLI Consortium 2002, 2004).

As discussed in the Introduction, an advantage of the multivariate methods over their univariate counterparts is the additional power afforded by the consideration of correlations between traits. In the multivariate genome screen seven loci yielded levels of linkage with $P<0.01$. 
Whilst the regions on chromosomes 16 and 19 had previously been identified by a univariate genome screen, the remaining five loci on chromosomes 1, 4, 5, 7 and 10 represented novel putative QTLs. However, within the extended wave 1 and 2 sample only the chromosome 16 linkage remained significant. Of the seven loci investigated those on chromosomes 1, 4, 5 and 7 showed a complete lack of linkage within the wave 1 and 2 combined sample. In these cases it is expected that the failure to replicate indicates the presence of a type I error (false positive). However, given that the chromosome 19 locus had previously been shown to be linked within these samples, it is unlikely that this region represents a false positive. Furthermore, even the chromosome 16 locus, which had previously been shown to be consistently linked to the NWR trait within samples of language impaired families, only just exceeded the $P=0.01$ threshold of linkage ( $P=0.008$ - Figure 2$)$, and permutations indicated that the nominal p-values may be over-conservative (see Supplementary Table 2). Thus, given that the linkage levels on chromosomes 10 and 19 continue to border a $P=0.01$ threshold within the wave 1 and 2 combined sample $(P=0.019$ and $P=0.017$ respectively), it remains credible that these two loci represent valid QTLs for language impairment. At these loci an alternative explanation for the lack of replication would be that the regions only contribute to linkage within a subset of families. Although all the samples used for this study were ascertained using identical selection criteria, they were collected from four different sites spread across the UK, each with their own individual research interests. The wave 1 samples were selected from a clinical group at Guy's Hospital, London and an epidemiological study in Cambridge, whilst wave 2 consisted, for the greater part, of samples from a clinical investigation of receptive Language Impairments in Edinburgh, supplemented with samples from Guy's, Cambridge and a small clinical study in Aberdeen. Thus, for example, if the loci on chromosomes 10 and 19 contributed to SLI susceptibility within the less severely affected, epidemiological (Cambridge) families alone, or within populations from the South of England, one may predict that they would not be replicated so strongly within the wave 2 samples. Interestingly, on chromsome 19, when one considered the epidemiological (Cambridge) and Clinical (Guy's and Aberdeen and
Edinburgh) samples separately, the evidence for linkage to the expressive language score was much stronger in the epidemiological cohort $(P=0.000996)$ than the clinical samples $(P=0.0612)$.

Previous research has shown that the power of multivariate linkage analysis is at its strongest when the QTL and residual trait correlations have opposite signs (Amos et al. 2001; Evans, 2002). The QTL negative correlation can be represented at each peak by performing a univariate analysis on the QTL factor score, where the factor loadings are determined by the QTL parameters in the multivariate analysis. When considering ELS, RLS and NWR, the chromosome 10 linkage region consisted of a negative QTL factor loading on NWR and positive QTL factor loadings on ELS and RLS, indicating that this locus may be related to a discrepancy between NWR and the CELF measures, interpretable as a language score on which some adjustment for phonological competence has been made.

Our initial strategy had been to perform a detailed follow-up investigation of any locus which consistently showed linkage above $P=0.01$ within the three-trait (ELS, RLS and NWR) model applied to the wave 1 and wave 1 and 2 combined samples. However, the multivariate analyses demonstrated that this was only true for a single region on chromosome 16. Thus, we instead chose to apply the follow up model to both of the regions which had previously been shown to be of importance in SLI (i.e. chromosomes 16 (SLI1) and 19 (SLI2)). The comprehensive model applied in these regions made use of the complete dataset available, comprising 178 families and eleven traits (CELF subtests Oral Directions (OD), Formulating Sentences (FSENT), Recalling Sentences (RSENT), Word Classes (WC), Semantic Relations (SREL) and Sentence Assembly (SA), WORD subtests Basic Reading (BRSS), Spelling (SPSS) and Reading Comprehension (RCSS), Non-Word Repetition (NWR) and Performance IQ (PIQ)). The sheer number of traits in this dataset had previously precluded the direct use of the CELF subtests, which may be expected to provide cleaner measures than the composite ELS and RLS scores. Thus it was hoped that the multivariate modelling of these traits would allow a better evaluation of which linguistic processes are important in the development of SLI than would be allowed by univariate analysis alone. 
Our initial multivariate study of chromosome 16 using the combined waves 1 and 2 dataset implied that two traits were significant across SLI1: oral directions in the Cambridge cohort (qOD_Cam) and NWR in the combined sample. However, by considering the underlying QTL parameter estimates it became clear that the SLI1 effect on NWR was substantially stronger than that on OD. By incorporating the WORD literacy measures (Rust et al. 1993) into the model, together with the two phenotypes above, we could determine that the larger SLI1 influences were shared between NWR, singleword reading (BRSS) and single-word spelling (SPSS). Other studies have also suggested shared genetic influences between NWR and literacy tests. Bishop (2001) used bivariate DeFries-Fulker analysis to examine the genetic relationship between NWR and literacy tests (reading and spelling tests taken from the British Ability Scales (Elliott et al. 1983) and the Vernon Graded Word Spelling test (Vernon, 1977), respectively. The bivariate heritabilities were significant for both NWR and reading, and NWR and spelling, with estimates of 0.864 and 0.700 , respectively, before adjusting for IQ, implying that genes affecting NWR may also influence literacy abilities. Hsu et al. (2002) performed reciprocal aggregation analysis on various reading, spelling and language measures, by estimating parent-offspring and siblingsibling correlations for each measure while using a separate measure as a covariate. This approach suggested that non-word memory, NWM (Wagner et al. 1999), accounts for a large proportion of the familial pattern of spelling, taken from the Wide Range Achievement Test, WRAT-3 (Wilkinson, 1993), implying that phonological short-term memory may have some shared genetic influences with spelling ability. In addition, Catts (1993) indicated that phonological measures seemed to be the best predictors for word recognition (single-word reading), whereas semantic-syntactic language abilities, such as the traits measured in CELF, accounted for a larger proportion of the variation in reading comprehension. Although the SLIC univariate investigations found a LOD score of 1.99 for the WORD reading comprehension score (RCSS) on chromosome 16q (The SLI Consortium, 2004), the multivariate analysis indicated that this linkage could be explained by correlations between NWR, BRSS and SPSS. Therefore RCSS was not found to be significant in the multivariate study, again suggesting that SLI1 may influence phonological short-term memory.

Previous univariate studies on chromosome 19 found linkage across the two waves of families but with two separate measures. In wave 1 the linkage on chromosome 19 appeared to be specific to the ELS trait, with no evidence for linkage to NWR or RLS (The SLI Consortium, 2002). In wave 2 , however, the converse appeared to be true, with suggestive levels of linkage to NWR but only nominal levels of linkage to ELS and RLS (The SLI Consortium, 2004). Both of these univariate linkages were characterised by broad peaks which spanned the majority of the q arm of chromosome 19. The multivariate analyses confirmed linkage at SLI2, and reflected the findings of the univariate analyses in that two distinct linkages were found in this region. The proximal multivariate linkage coincided with the univariate peak at $106 \mathrm{cM}$, where linkage was strongest to the ELS trait within the wave 1 samples. At this locus linkage could be equally attributed to each of the six CELF subtests (both receptive (OD, WC, SREL) and expressive (FSENT, RSENT and SA)). The second, distal multivariate locus at $174 \mathrm{cM}$ overlapped with the telomeric end of the univariate peak, in a region where linkage had previously been demonstrated with the NWR trait in the Edinburgh cohort. In contrast to the proximal locus, the multivariate linkage in this region could be attributed to just two CELF subtests, SREL (receptive) and RSENT (expressive), and to a lesser extent NWR. Thus, since different traits contributed to each of the multivariate linkages on chromosome 19, one may postulate that the divergence of the univariate results may be explained by the existence of two adjacent loci on chromosome 19. Alternatively, a single gene variant may underlie the linkage on chromosome 19. Under such a hypothesis we would expect this gene to influence a fundamental linguistic process, and therefore be reflected in a range of deficits across multiple measures in different groups.

In conclusion, this study uses multivariate linkage techniques to extend previous univariate studies of a sample of families affected by Specific Language Impairment (SLI). This investigation has provided support for previously identified loci on chromosomes 16 and 19, and allowed an exploration of the interaction between additional phenotypes in these regions. 


\section{Acknowledgments}

First, we would like to thank all the families who have participated in the study and the professionals who continue to make this study possible. We thank everyone at the Newcomen, CLASP, Edinburgh, and Aberdeen centres for their involvement in the project, Leila Jannoun, Jane Addison, Clare Craven, Deborah Jones, Tilly Storr, Til Utting-Brown, Margaret Main, Judith Steel and Alan MacLean for their assistance with data collection and management; and Angela Marlow for advice on the analysis. All laboratory work and the collection of families from Guy's Hospital was funded by the Wellcome Trust. CLASP is funded by the Wellcome Trust, British Telecom, the Isaac Newton Trust, an NHS Anglia \& Oxford Regional R\&D Strategic Investment Award, and an NHS Eastern Region R\&D Training Fellowship Award. The Edinburgh group was supported by the Chief Scientist's Office, Scotland. The Aberdeen group was supported by grants from Grampian Healthcare Trust and Grampian Primary Care NHS Trust. A.P.M., L.R.C. and D.V.M.B. are Wellcome Trust Principal Research Fellows. S.E.F. is a Royal Society Research Fellow.

\section{References}

Almasy, L. \& Blangero, J. (1998) Multipoint quantitative-trait linkage analysis in general pedigrees. Am J Hum Genet 62, 1198-1211.

Amos, C. I. \& de Andrade, M. (2001) Genetic linkage methods for quantitative traits. Stat Methods Med Res 10, 3-25.

Amos, C. I., de Andrade, M. \& Zhu, D. K. (2001) Comparison of multivariate tests for genetic linkage. Hum Hered 51, 133-144.

Bartlett, C. W., Flax, J. F., Logue, M. W., Vieland, V. J., Bassett, A. S., Tallal, P. \& Brzustowicz, L. M. (2002) A major susceptibility locus for specific language impairment is located on 13q21. Am J Hum Genet 71, 45-55.

Bartlett, C. W., Flax, J. F., Logue, M. W., Smith, B. J., Vieland, V. J., Tallal, P. \& Brzustowicz, L. M. (2004) Examination of potential overlap in autism and language loci on chromosomes 2, 7, and 13 in two independent samples ascertained for specific language impairment. Hum Hered 57, 10-20.

Beitchman, J. H. \& Inglis, A. (1991) The continuum of linguistic dysfunction from pervasive developmental disorders to dyslexia. Psychiatr Clin North Am 14, 95-111.

Bishop, D. V. M. \& Edmundson, A. (1986) Is otitis media a major cause of specific developmental language disorders? Br J Comm Disord 21, 321-338.

Bishop, D. V. M., North, T. \& Donlan, C. (1995) Genetic basis of specific language impairment: evidence from a twin study. Dev Med Child Neurol 37, 56-71.

Bishop, D. V. M., North, T. \& Donlan, C. (1996) Nonword repetition as a behavioural marker for inherited language impairment: evidence from a twin study. J Child Psychol Psychiatry 37, 391-403.

Bishop, D. V. M. (2001) Genetic influences on language impairment and literacy problems in children : same or different? J Child Psychol Psychiatry 42, 189-198.

Bishop, D. V. M. \& Snowling, M. J. (2004) Developmental dyslexia and specific language impairment: same or different? Psychol Bull 130, 858-886.

Boomsma, D. I. \& Dolan, C. V. (1998) A comparison of power to detect a QTL in sib-pair data using multivariate phenotypes, mean phenotypes, and factor scores. Behav Genet 28, 329-340.

Catts, H. W. (1993) The relationship between speechlanguage impairments and reading disabilities. J Speech Hear Res 36, 948-958.

DeFries, J. C. \& Fulker, D. W. (1985) Multiple Regression Analysis of Twin Data. Behav Genet 15, 467-473.

Elliott, C., Murray, D. J. \& Pearson, L. S. (1983) British ability scales. NFER-Nelson, Windsor, UK.

Evans, D. M. (2002) The power of multivariate quantitativetrait loci linkage analysis is influenced by the correlation between variables. Am J Hum Genet 70, 1599-1602.

Fisher, S. E. \& DeFries, J. C. (2002) Developmental dyslexia: Genetic dissection of a complex cognitive trait. Nat Rev Neuro 3, 767-780.

Gathercole, S. E., Willis, C. S., Baddeley, A. D. \& Emslie, H. (1994) The children's test of nonword repetition: a test of phonological working memory. Memory 2, 103-127.

Hsu, L., Wijsman, E. M., Berninger, V. W., Thomson, J. B. \& Raskind, W. H. (2002) Familial aggregation of dyslexia phenotypes. II: Paired correlated measures. Am J Med Genet B Neuropsychiatr Genet 114, 471-478.

Kruglyak, L., Daly, M. J., Reeve-Daly, M. P. \& Lander, E. S. (1996) Parametric and nonparametric linkage analysis: a unified multipoint approach. Am J Hum Genet 58, 13471363.

Law, J., Boyle, J., Harris, F., Harkness, A. \& Nye, C. (1998) Screening for speech and language delay: A systematic review of the literature. Health Technol Assess 2, 1-184.

Lewis, B. A. \& Thompson, L. A. (1992) A study of developmental speech and language disorders in twins. J Speech Hear Res 35, 1086-1094.

Lewis, B. A., Freebairn, L. A. \& Taylor, H. G. (2000) Academic outcomes in children with histories of speech sound disorders. J Commun Disord 33, 11-30.

Marlow, A. J., Fisher, S. E., Francks, C., MacPhie, I. L., Cherny, S. S., Richardson, A. J., Talcott, J. B., Stein, J. F., Monaco, A. P. \& Cardon, L. R. (2003) Use of multivariate linkage analysis for dissection of a complex cognitive trait. Am J Hum Genet 72, 561-570.

Neale, M. C., Boker, S. M., Xie, G. \& Maes, H. H. (2002) Mx: Statistical Modelling. Department of Psychiatry, VCU Box 900126, Richmond, VA, 23298. 
Neils, J. \& Aram, D. M. (1986) Family history of children with developmental language disorders. Percep Mot Skills 63, 655-658.

O'Grady, W. (2005) How Children Learn Language. Cambridge: Cambridge University Press.

Raitano, N. A., Pennington, B. F., Tunick, R. A., Boada, R. \& Shriberg, L. D. (2004) Pre-literacy skills of subgroups of children with speech sound disorders. J Child Psychol Psychiatry 45, 821-835.

Rust, J., Golombok, S. \& Trickey, G. (1993) Wechsler Objective Reading Dimensions. Psychological Corporation, Sidcup, Kent, UK.

Schmitz, S., Cherny, S. S. \& Fulker, D. W. (1998) Increase in power through multivariate analyses. Behav Genet 28, 357-363.

Self, S. G. \& Liang, K. (1987) Asymptotic properties of maximum likelihood estimators and likelihood ratio tests under nonstandard conditions. Journal Am Stat Assoc 82, 605-610.

Semel, E. M., Wiig, E. H. \& Secord, W. (1992) Clinical Evaluation of Language Fundamental, Revised. Psychological Corporation, San Antonio.

Smith, S. D., Pennington, B. F., Boada, R. \& Shriberg, L. D. (2005) Linkage of Speech Sound Disorder to reading diability loci. J Child Psychol Psychiatry 46, 1057-1066.

Stein, C. M., Schick, J. H., Taylor, G., Shriberg, L. D., Millard, C., Kundtz-Kluge, A., Russo, K., Minich, N., Hansen, A., Freebairn, L. A., Elston, R. C., Lewis, B. A. \& Iyengar, S. K. (2004) Pleiotropic effects of a chromosome 3 locus on speech-sound disorder and reading. Am J Hum Genet 74, 283-297.

Stein, C. M., Millard, C., Kluge, A., Miscimarra, L. E., Cartier, K. C., Freebairn, L. A., Hansen, A. J., Shriberg, L. D., Taylor, H. G., Lewis, B. A. \& Iyengar, S. K. (2006) Speech Sound Disorder Influenced by a Locus in $15 \mathrm{q} 14$ Region. Behav Genet 36, 858-868.

Stromswold, K. (1998) Genetics of Spoken Language Disorders. Human Biology 70, 297-324.

Tallal, P., Ross, R. \& Curtiss, S. (1989) Familial aggregation in specific language impairment. J Speech Hear Disord 54, 167-173.
The SLI Consortium (2002) A genomewide scan identifies two novel loci involved in specific language impairment. Am J Hum Genet 70, 384-398.

The SLI Consortium (2004) Highly significant linkage to the SLI1 locus in an expanded sample of individuals affected by specific language impairment. Am J Hum Genet 74, 12251238.

Tomblin, J. B., Records, N. L., Buckwalter, P., Zhang, X., Smith, E. \& O'Brien, M. (1997) Prevalence of specific language impairment in kindergarten children. J Speech Lang Hear Res 40, 1245-1260.

Tomblin, J. B. \& Buckwalter, P. R. (1998) Heritability of poor language achievement among twins. J Speech Lang Hear Res 41, 118-199.

Vernon, P. E. (1977) Graded Word Spelling Test. Hodder \& Stoughton, London.

Viding, E., Spinath, F. M., Price, T. S., Bishop, D. V. M., Dale, P. S. \& Plomin, R. (2004) Genetic and environmental influence on language impairment in 4-year-old same-sex and opposite-sex twins. J Child Psychol Psychiatry 45, 315325.

Vogler, G. P., Tang, W., Nelson, T. L., Hofer, S. M., Grant, J. D., Tarantino, L. M. \& Fernandez, J. R. (1997) A multivariate model for the analysis of sibship covariance structure using marker information and multiple quantitative traits. Genet Epidemiol 14, 921-926.

Wang, T. \& Elston, R. C. (2007) Regression-based multivariate linkage analysis with an application to blood pressure and body mass index. Ann Hum Genet 71, 96-106.

Wagner, R., Torgesen, J. \& Rashotte, C. A. (1999) Comprehensive test of phonological processing (CTOPP). Pro-Ed, Austin, TX.

Wechsler, D. (1992) Wechsler intelligence scale for children. 3rd edn. The Psychological Corporation, 555 Academic Court, San Antonio, TX, 78204-2498.

Wilkinson, G. (1993) Wide range achievement test - third edition (WRAT-3). Wide Range, Wilmington, DE.

Accepted: 23 October 2006

Received: 23 February 2007 